\title{
Risk factors for postoperative pneumonia after cardiac surgery: a prediction model
}

\author{
Dashuai Wang, Xiaofan Huang, Hongfei Wang, Sheng Le, Han Yang, Feng Wang, Xinling Du \\ Department of Cardiovascular Surgery, Union Hospital, Tongji Medical College, Huazhong University of Science and Technology, Wuhan, China \\ Contributions: (I) Conception and design: X Du, X Huang, D Wang; (II) Administrative support: X Du, H Wang, S Le; (III) Provision of study \\ materials or patients: X Du, X Huang, H Wang; (IV) Collection and assembly of data: D Wang, H Yang, F Wang, H Wang; (V) Data analysis and \\ interpretation: D Wang, S Le, X Huang; (VI) Manuscript writing: All authors; (VII) Final approval of manuscript: All authors. \\ Correspondence to: Xiaofan Huang, MD; Xinling Du, MD, PhD. Union Hospital, Wuhan Jiefang Road, No. 1277, Wuhan 430022, China. \\ Email: Dr_xfhuang@hust.edu.cn; xinlingdu@hust.edu.cn.
}

\begin{abstract}
Background: Postoperative pneumonia is the main infectious complication following cardiac surgery and is associated with significant increases in morbidity, mortality and health care costs. The aim of this study was to identify potential risk factors related to the occurrence of postoperative pneumonia in adult patients undergoing cardiac surgery and to develop a predictive system.

Methods: Adult patients who underwent open heart surgery in our institution between 2016 and 2019 were enrolled in this study. Preoperative and intraoperative variables were collected and analyzed. A multivariate prediction model for evaluating the risk of postoperative pneumonia was established using logistic regression analysis via forward stepwise selection, and points were assigned to significant risk factors based on their regression coefficient values.

Results: Postoperative pneumonia occurred in 530 of the 5,323 patients $(9.96 \%)$. Prolonged stays in the postoperative intensive care unit (ICU) and hospital, as well as higher mortality (25.66\% versus $0.65 \%$ ), were observed in patients with postoperative pneumonia. Multivariate analysis identified 13 independent risk factors including patient demographics, comorbidities, cardiac function, cardiopulmonary bypass (CPB) duration, and blood transfusion. The prediction model showed good discrimination (C-statistic: 0.80$)$ and was well calibrated (Hosmer-Lemeshow $\chi^{2}=7.907, \mathrm{P}$ value $=0.443$ ). A 32-point risk score was generated, and then three risk intervals were defined.

Conclusions: We derived and validated a prediction model for postoperative pneumonia after cardiac surgery incorporating 13 easily discernible risk factors. The scoring system may be helpful for individualized risk estimations and clinical decision-making.
\end{abstract}

Keywords: Postoperative pneumonia; cardiac surgery; risk factor; prediction model; risk score

Submitted Dec 27, 2020. Accepted for publication Mar 05, 2021.

doi: $10.21037 /$ jtd-20-3586

View this article at: http://dx.doi.org/10.21037/jtd-20-3586

\section{Introduction}

Postoperative pneumonia is the most common infection following cardiac surgery and is strongly related to increased risks of morbidity, mortality, prolonged intensive care unit (ICU) and hospital stay $(1,2)$. Consequently, resource utilization and total treatment costs are also significantly augmented $(3,4)$. The incidence of postoperative pneumonia differs substantially in various studies, with reported rates of $2.1-21.6 \%(5,6)$, and evidence suggests that the real incidence may be underreported (7).

The characteristics of patients undergoing cardiac surgery have changed over the years. Despite considerable progress in surgery and anesthesia, increasing aging populations with multiple comorbidities and the emergence of antibiotic-resistant pathogens have obviously increased 
the proportion of patients at a higher risk for postoperative pneumonia (8-10). Several risk factors for pneumonia after cardiac surgery have been identified, such as a reduced pulmonary reserve and poor cardiac function. However, numerous studies were based on small samples and narrow patient selection, and most published literature were nearly a decade old or more (5,11-13). Furthermore, the vast majority of published studies were conducted in developed countries, especially in the United States and Europe. Studies from developing countries that make up the majority of the world's population are rare. Risk factors for postoperative pneumonia following cardiac surgery may vary with relationship to the medical background of the patient. Thus, more evidence on risk factors for pneumonia after cardiac surgery is still needed.

The aim of this study was to identify potential predictors for the development of pneumonia in patients undergoing cardiac surgery and to derive and validate a risk prediction model to decrease the incidence of postoperative pneumonia by reasonable prevention and effective treatment.

We present the following article in accordance with the STROBE reporting checklist (available at http://dx.doi. org/10.21037/jtd-20-3586).

\section{Methods}

\section{Ethical statement}

The study was conducted in accordance with the Declaration of Helsinki (as revised in 2013). The study was approved by the Ethics Committee of Tongji Medical College of Huazhong University of Science and Technology (IORG No. IORG0003571) and individual consent for this retrospective analysis was waived.

\section{Study population}

We conducted a retrospective, observational study including consecutive adult patients following open heart surgery between January 2016 and December 2019 at our institution, a tertiary care center located in Wuhan, China. All patients were ventilated using a lung protective ventilation strategy (tidal volume was $6-8 \mathrm{~mL} / \mathrm{kg}$, driving pressure was $15 \mathrm{cmH}_{2} \mathrm{O}$, and positive end-expiratory pressure was $5 \mathrm{cmH}_{2} \mathrm{O}$ ). Patients with the following conditions were excluded from this study: (I) acquired pneumonia within 2 weeks prior to surgery; (II) organ transplantation, immune deficiency, or immunosuppression;
(III) death or discharge within 48 hours after surgery; and (IV) incomplete medical record data.

\section{Data collection}

Clinical data were collected using the electronic medical records management system of the hospital. Preoperative variables included demographics [sex, age, height, weight, body mass index (BMI), history of smoking and alcohol consumption], comorbidities [hypertension, diabetes mellitus, chronic obstructive pulmonary disease (COPD), cerebrovascular disease, peripheral vascular disease, renal disease, atrial fibrillation, gastrointestinal tract disease, general and cardiac surgical history], cardiac anatomy and function [New York Heart Association (NYHA) class, left ventricular ejection fraction, and the diameters of the four chambers], and laboratory values. Intraoperative variables included surgical type, blood transfusion, cardiopulmonary bypass (CPB) time, and aortic cross clamp time. Postoperative variables included the lengths of mechanical ventilation, ICU and hospital stay, reintubation, tracheotomy, mortality, and etiological examination.

A history of smoking was defined as current or previous daily smoking. A history of alcohol consumption (consumption $>20 \mathrm{~g} /$ day or $>140 \mathrm{~g} /$ week) was defined as alcohol consumption at least once a week over a year, current alcohol consumption, or quitting for less than three years. Hypertension was defined as blood pressure $\geq 140 / 90 \mathrm{mmHg}$, previous diagnosis of hypertension, or use of antihypertensive medication. Diabetes mellitus was defined as fasting glucose $\geq 7.0 \mathrm{mmol} / \mathrm{L}$, random glucose $\geq 11.1 \mathrm{mmol} / \mathrm{L}$, previous diagnosis of diabetes mellitus, or use of diabetic medication. COPD was defined as FEV1/ FVC $\leq 0.7$. Renal insufficiency was defined as serum creatinine $>110 \mu \mathrm{mol} / \mathrm{L}$ or previous diagnosis of renal insufficiency. Hypoalbuminemia was defined as albumin $<35 \mathrm{~g} / \mathrm{L}$. Anemia was defined as hemoglobin $<120 \mathrm{~g} / \mathrm{L}$ for men or $<110 \mathrm{~g} / \mathrm{L}$ for women. BMI was calculated as weight in kilograms divided by the square of the height in meters.

\section{Definition}

The diagnostic criteria for pneumonia were defined according to the American guidelines $(14,15)$. In this study, postoperative pneumonia was considered clinically present when new and/or progressive pulmonary infiltrates were identified on chest radiographs or computed tomography in conjunction with two or more of the following criteria: fever $\left(>38^{\circ} \mathrm{C}\right)$ without other identifiable causes, leukocytosis 
$\left(>12 \times 10^{9} / \mathrm{L}\right)$ or leukopenia $\left(<4 \times 10^{9} / \mathrm{L}\right)$, and purulent secretions. The microbiological etiology of postoperative pneumonia was mainly identified by semiquantitative cultures from endotracheal aspiration of lower respiratory tract secretions or sputum with an initial microscopic examination combined with quantitative bacterial cultures.

\section{Statistical analysis}

Statistical analysis was performed using SPSS (IBM SPSS Statistics, version 24). Univariate analysis was first conducted to initially screen possible risk factors for pneumonia after cardiac surgery. Continuous variables were expressed as means \pm standard deviations and analyzed by Student's $t$-test when normally distributed. Those that were skewed were presented as medians (Q1, Q3) and compared by nonparametric tests. Categorical variables were presented as counts with percentages and analyzed by the chi-square test or Fisher's exact test. Factors screened by univariate analysis were then entered as candidate variables into a forward stepwise multivariate logistic regression model to identify independent risk factors. A simplified point-based prediction rule was developed from the regression model with each regression coefficient divided by the smallest coefficient and rounded to the nearest integer to obtain the weighted value. Continuous variables were dichotomized in the final model based on clinical relevance, cutoff values used in previous studies, and statistical significance, to assign risk points and achieve better clinical application. A composite risk score was then generated by the sum of individual risk points. The calibration of the model was evaluated using the HosmerLemeshow test for goodness of fit and the discrimination was assessed through the area under the receiver operating characteristic curve. We internally validated the model by bootstrapping using 1,000 replications. Bootstrapping allows internal validation of prediction models by repeated sampling with replacement from the original dataset. Survival analysis adopted Kaplan-Meier method. A consort diagram has been provided (Figure 1).

\section{Results}

\section{Demographic characteristic}

A total of 5,323 adult patients who underwent cardiac surgery were enrolled in this study including 3,001 males. The mean age was $51.18 \pm 12.92$ years. The incidence of postoperative pneumonia was $9.96 \%$, which mainly developed in the first postoperative week (87\%), with a median time of $4[2,5]$ days (Figure S1).

Of the 5,323 operations, more than half (2,897 cases) were performed for isolated valve replacement or repair, $11 \%$ (591 cases) for isolated coronary artery bypass grafting (CABG), 9\% (481 cases) for mixed valve surgery and CABG, 17\% (904 cases) for aortic surgery, and 8\% (450 cases) for other types. Blood products were used in $83 \%$ (4,401 cases) of the procedures, and the median duration of $\mathrm{CPB}$ was $105[78,142]$ minutes.

The patients constituting this study population had different medical histories and underlying diseases. According to the NYHA class, 876 patients (16\%) were considered class III-IV. A history of smoking was noted in 1,548 patients (29\%), alcohol consumption in 1,174 patients (22\%), previous cardiac surgery in 376 patients $(7 \%)$, hypertension in 1,604 patients (30\%), diabetes mellitus in 438 patients (8\%), COPD in 557 patients $(10 \%)$, atrial fibrillation in 912 patients (17\%), renal insufficiency in 524 patients $(10 \%)$, and pulmonary artery hypertension in 1,390 patients $(26 \%)$.

\section{Risk factors and the prediction model}

Univariate analysis was used to analyze potential risk factors for the development of postoperative pneumonia and showed that many aspects, including demographics, comorbidities, cardiac anatomy and function, laboratory values, operative time, and blood transfusion, were significantly different between patients with and without postoperative pneumonia (Table 1).

In the multivariate analysis, 13 independent risk factors for postoperative pneumonia were identified, including age $>60$ years, hypertension, diabetes mellitus, smoking history, COPD, BMI $\geq 24 \mathrm{~kg} / \mathrm{m}^{2}$, renal insufficiency, heart surgery history, NYHA class III-IV, preoperative anemia, hypoalbuminemia, CPB time $>120$ minutes and blood transfusion (Table 2). Odds ratios with corresponding $95 \%$ confidence intervals (CIs) and assigned points are presented. A simplified point-based risk score used to predict the risk of postoperative pneumonia was then generated (Figure 2). There were 32 possible points in the composite risk index, with scores ranging from 0 to 27 in this study with a median of $9[6,13]$. The predicted rate of postoperative pneumonia ranged from $0.61 \%$ for those with a score of 0 , to $93.4 \%$ for those with a score of 32 .

The occurrence of pneumonia after cardiac surgery could be accurately predicted with the model as the 


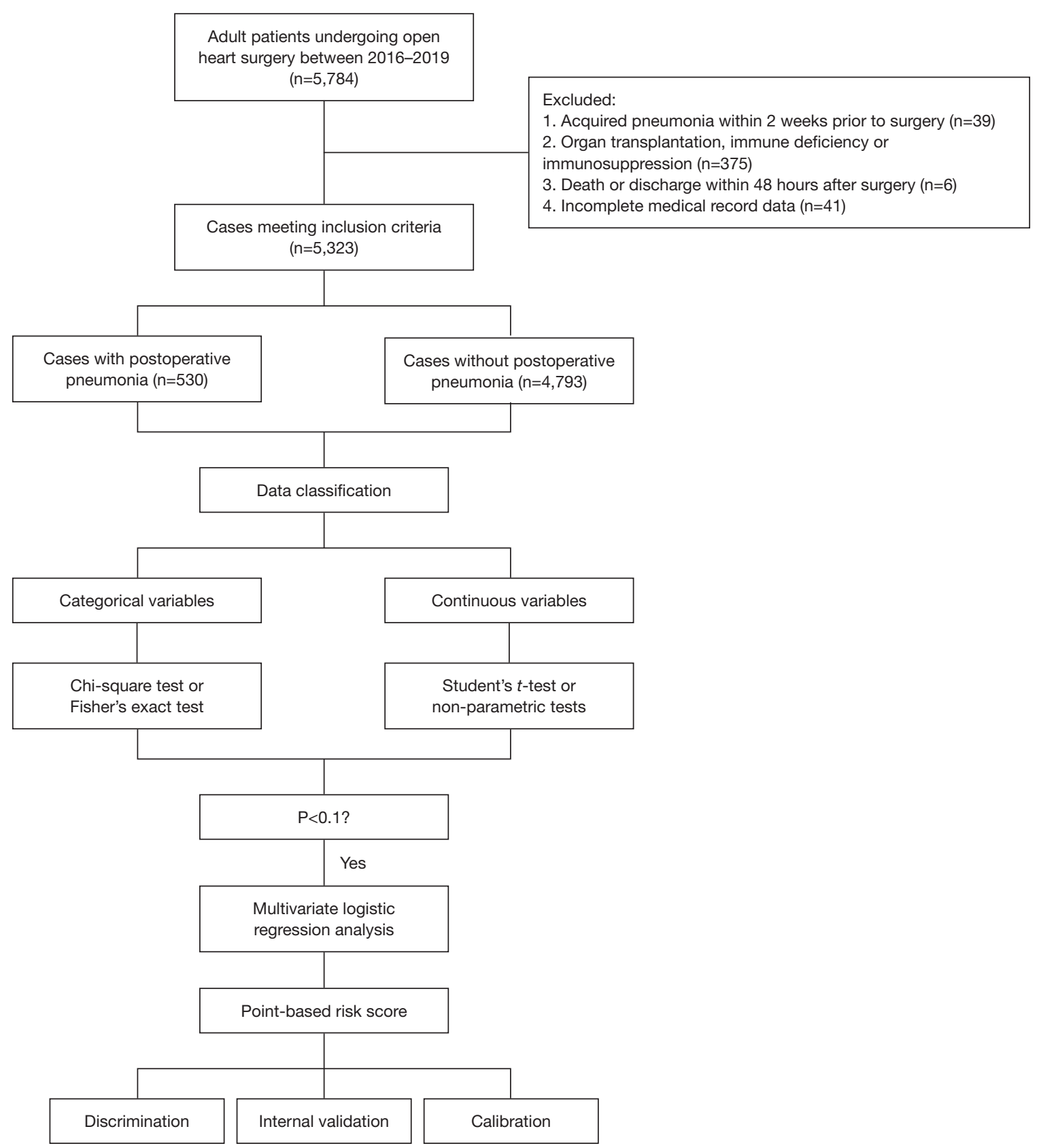

Figure 1 Consort diagram of the study. The flow of subjects from enrollment to data analysis is shown.

C-statistic was 0.80 (95\% CI, 0.79-0.82), demonstrating good discrimination. The correlation between the expected and observed numbers of postoperative pneumonia events was high $(\mathrm{r}=0.99)$, indicating good calibration (HosmerLemeshow $\chi^{2}=7.907$, $\mathrm{P}$ value $\left.=0.443\right)$. The calibration was also good by visual inspection. Our risk score outperformed Kilic's risk score (C-statistic: 0.69; 95\% CI, 0.67-0.71) and
Allou's risk score (C-statistic: 0.57; 95\% CI, 0.54-0.60) in predicting postoperative pneumonia $(\mathrm{P}<0.001$; Figure 3$)$. We also internally validated the discrimination of this model using the bootstrapping method (1,000 replications). Finally, three optimal risk intervals were identified as low (<11 points), medium (11-15 points) and high risk ( $\geq 16$ points) for postoperative pneumonia according to the 
Table 1 Univariate analysis of possible risk factors for postoperative pneumonia in patients undergoing cardiac surgery

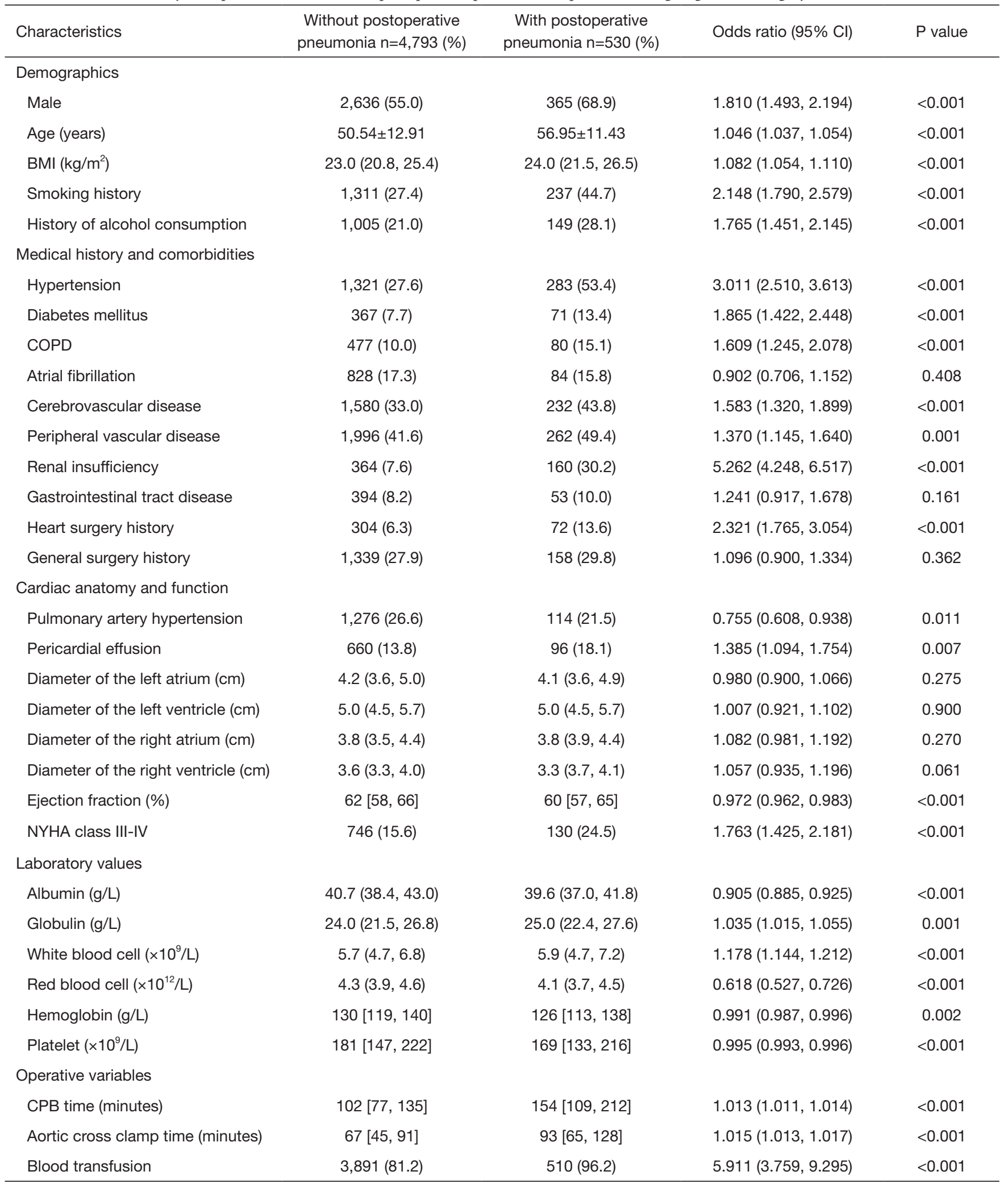

$\mathrm{BMI}$, body mass index; $\mathrm{Cl}$, confidence interval; COPD, chronic obstructive pulmonary disease; CPB, cardiopulmonary bypass; NYHA, New York Heart Association. 
Table 2 Multivariate analysis of independent risk factors for postoperative pneumonia in patients undergoing cardiac surgery, with corresponding point values

\begin{tabular}{|c|c|c|c|c|}
\hline Characteristics & Odds ratio (95\% Cl) & $P$ value & Coefficient (B) & Point value \\
\hline Hypertension & 1.787 (1.447-2.208) & $<0.001$ & 0.581 & 2 \\
\hline Diabetes mellitus & $1.378(1.015-1.871)$ & 0.040 & 0.321 & 1 \\
\hline Smoking history & $1.689(1.380-2.068)$ & $<0.001$ & 0.524 & 2 \\
\hline $\mathrm{BMI} \geq 24 \mathrm{~kg} / \mathrm{m}^{2}$ & $1.274(1.039-1.562)$ & 0.020 & 0.242 & 1 \\
\hline Renal insufficiency & $2.670(2.095-3.402)$ & $<0.001$ & 0.982 & 4 \\
\hline Heart surgery history & $2.544(1.872-3.456)$ & $<0.001$ & 0.934 & 4 \\
\hline NYHA class III-IV & $1.447(1.146-1.826)$ & 0.002 & 0.369 & 2 \\
\hline CPB time $>120$ minutes & $2.622(2.133-3.223)$ & $<0.001$ & 0.964 & 4 \\
\hline Blood transfusion & $3.531(2.212-5.636)$ & $<0.001$ & 1.262 & 5 \\
\hline Constant & 0.006 & $<0.001$ & -5.094 & \\
\hline
\end{tabular}

$\mathrm{BMI}$, body mass index; $\mathrm{Cl}$, confidence interval; COPD, chronic obstructive pulmonary disease; CPB, cardiopulmonary bypass; NYHA, New York Heart Association.

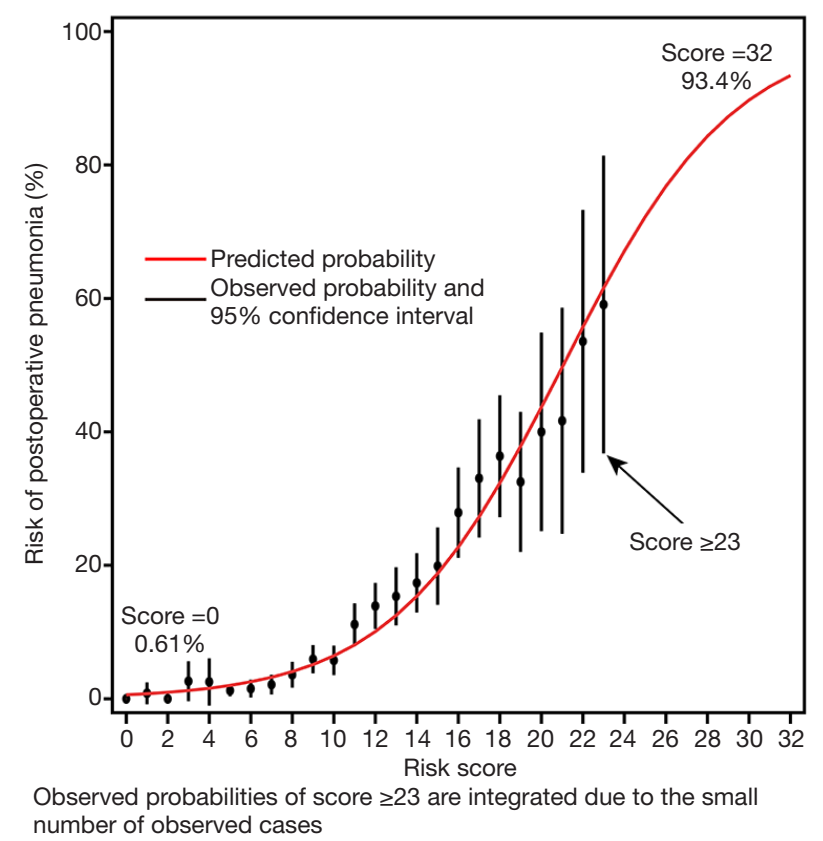

Figure 2 Predicted risk of postoperative pneumonia in adult patients who underwent cardiac surgery based on the 32-point risk score and the comparison with the observed probabilities.

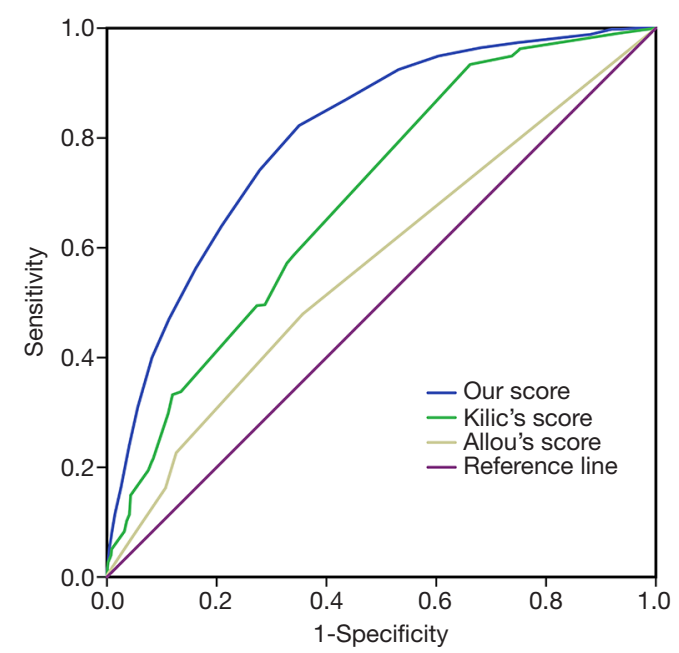

Figure 3 Receiver operating characteristic curve drawn using the 32-point risk score (C-statistic: 0.80; 95\% CI, 0.79-0.82) and the comparison with Kilic's risk score (C-statistic: 0.69; 95\% CI, 0.67-0.71) and Allou's risk score (C-statistic: 0.57; 95\% CI, 0.54 $0.60)$. The 32-point score outperformed both of the two models $(\mathrm{P}<0.001)$. CI, confidence interval. 
Table 3 Postoperative pneumonia risk score: distribution of patients and rates by intervals

\begin{tabular}{lccr}
\hline Risk intervals & Low risk $(<11$ points $)$ & Medium risk (11-15 points) & High risk $(\geq 16$ points $)$ \\
\hline No. $(\%)$ & $3,209(60.29)$ & $1,509(28.35)$ & $605(11.36)$ \\
Rate, \% $(95 \% \mathrm{Cl})$ & $2.93(2.35-3.51)$ & $14.84(13.05-16.64)$ & $35.04(31.23-38.85)$ \\
\hline
\end{tabular}

$\mathrm{Cl}$, confidence interval.

Table 4 Pathogenic microorganisms isolated from patients with postoperative pneumonia

\begin{tabular}{lc}
\hline Microorganisms & No. $\mathrm{n}=825(\%)$ \\
\hline Acinetobacter baumannii & $305(36.97)$ \\
Klebsiella pneumoniae & $157(19.03)$ \\
Pseudomonas aeruginosa & $91(11.03)$ \\
Staphylococcus aureus & $135(16.36)$ \\
Other bacteria & $132(16.0)$ \\
Fungi & $5(0.61)$ \\
Polymicrobial & $182(34.34)$ \\
\hline
\end{tabular}

simplified risk score and clinical practice (Table 3).

\section{Etiology}

The microorganisms isolated and cultured from patients with postoperative pneumonia are summarized in Table 4 . Acinetobacter baumannii and Klebsiella pneumoniae were the most common pathogens, followed by Staphylococcus aureus and Pseudomonas aeruginosa. Many fungi were also isolated in the present study. Polymicrobial pneumonia (pneumonia due to two or more pathogens) was found in $34.34 \%$ of the patients.

\section{Outcome}

Mechanical ventilation was discontinued in $81 \%$ of the patients in the first two days after surgery, but the duration differed substantially between patients with and without postoperative pneumonia. The median time of mechanical ventilation in patients without postoperative pneumonia was $22[18,39]$ hours, and the removal rate of the endotracheal tube was $89 \%$ within the first two days. However, the corresponding values in patients with postoperative pneumonia were significantly different at $125[69,223]$ hours and $15 \%$, respectively. The overall mortality rate was $3.14 \%$ and increased significantly in patients with postoperative pneumonia (25.66\%). Obviously increased risks of reintubation, tracheotomy, prolonged ICU and hospital stay were also observed in patients with pneumonia compared to patients without that. Details of the comparison are presented in Table S1.

\section{Discussion}

Postoperative pneumonia has been well recognized as an important cause of morbidity, mortality and increased health care costs $(14,16)$, which was reaffirmed by the results of this study. The observed incidence of pneumonia in this study was $9.96 \%$, which falls within the previously reported range (17). Gram-negative bacteria predominated in the development of postoperative pneumonia, with $A$. baumannii, $K$. pneumoniae and $P$. aeruginosa being the most common species. Gram-positive organisms such as $S$. aureus and fungi such as C. albicans also played important roles, similar to previous findings (10). The overall mortality rate was similar to those reported in other studies $(18,19)$. However, mortality among patients with pneumonia was much higher than that among patients without pneumonia, which is consistent with published papers (2), emphasizing the need to identify risk factors and high-risk patients.

In this study, we used data from 5,323 patients who underwent cardiac surgery at our institution to derive and validate a multivariate prediction model for postoperative pneumonia. Thirteen readily obtainable risk factors were identified, and a simplified 32-point risk score was generated. Then, three risk intervals were identified as low-, medium- and high-risk.

Several prediction rules for pneumonia after cardiac surgery have been developed in the United States and Europe (6,9,20-22), but no one has been widely recognized so far. More evidence should be provided on risk factors for pneumonia after cardiac surgery, especially in developing countries. As one of the biggest developing countries, it is a pity that there is not yet a high-quality study in this area in China, whose population accounts for about one-fifth of the world's population. This work may contribute significantly as it's the first large-scale study to develop and validate a 
clinical risk score for postoperative pneumonia after cardiac surgery conducted in China.

Independent risk factors for pneumonia after cardiac surgery identified in different studies vary considerably, which may be attributed to differences in the population characteristics and the definition used for clinical diagnosis (1). Several patient characteristics and comorbidities identified as independent risk factors in our analysis have been reported in published literature. Strobel and colleagues conducted a prospective study among 16,084 consecutive patients at 33 centers in Michigan from 2011 to 2014 and developed a risk model (C-statistic: 0.74 ) for pneumonia after CABG utilizing 17 preoperative predictors (21). A $3.3 \%$ incidence of pneumonia was observed, and demographics and comorbidities, including advanced age, smoking, diabetes, low hematocrit, chronic lung disease and poor cardiac function, were considered relevant to an increased risk of postoperative pneumonia. Kilic and colleagues derived and validated a 33-point risk score for pneumonia after cardiac surgery in 6,222 patients, and 3 preoperative predictors (age $\geq 65$ years, chronic lung disease and peripheral vascular disease) were incorporated (20). The overall rate of postoperative pneumonia was $4.5 \%$, and the predictive ability was reliable in both the derivation dataset (C-statistic: 0.72 ) and the validation dataset (C-statistic: 0.76). Kinlin and colleagues identified smoking, COPD and high levels of serum creatinine as significant preoperative risk factors for pneumonia after CABG in their model (the C-statistics for derivation and validation datasets were 0.78 and 0.75 , respectively) among 17,143 patients at 32 hospitals in 6 states from 1999 to 2004 (6). Hortal and colleagues reported a predictive preoperative risk score for pneumonia after cardiac surgery in a prospective study among 1,844 patients between 2003 and 2006; the risk score comprised factors such as age $>70$ years, previous cardiac surgery, NYHA class IV, and a creatinine level $>1.5 \mathrm{mg} / \mathrm{dL}$ (9). The overall incidence of postoperative pneumonia in their study was $5.7 \%$, and in patients requiring mechanical ventilation for longer than 48 hours, the incidence increased to $45.9 \%$.

$\mathrm{CPB}$ is known to decrease pulmonary compliance, increase the likelihood of atelectasis, and contribute to pulmonary dysfunction by inducing systemic inflammatory responses and ischemia reperfusion injuries (23). As expected, a longer length of $\mathrm{CPB}$ was identified as an independent risk factor for postoperative pneumonia in our analysis, which is consistent with most existing research results (1). Allou and colleagues found that the duration of $\mathrm{CPB}$ was positively associated with the risk of postoperative pneumonia, and the risk increased 2.98-fold under the interaction of intraoperative transfusion of red blood cells and a CPB duration $>60$ minutes (24). Kilic and colleagues reported a 1.71-fold increase in the odds of pneumonia among patients who underwent CPB for more than 100 minutes (20). The use of minimally invasive CPB has been reported to be associated with improved clinical outcomes, and more efforts should be focused on the improvement of circulatory support techniques $(25,26)$.

Another independent risk factor identified in this study was blood transfusion. Although transfusion of blood products during cardiac surgery is a common practice and can be lifesaving, increasing evidence indicates that blood transfusion can have adverse clinical effects (27-30). Likosky and colleagues conducted a prospective, contemporaneous cohort study to explore the relationship between blood transfusion and pneumonia after CABG among 16,182 patients at 33 medical centers in Michigan from 2011 to 2013. The results showed that patients receiving blood transfusion had 3.4-fold increased odds of postoperative pneumonia, and these odds increased substantially with each additional unit of red blood cells transfused (31). Another study reported that limiting perioperative blood transfusion not only decreased the risk of adverse postoperative events such as postoperative pneumonia and death but also enormously reduced health care costs, and restricted blood transfusion was implemented by LaPar and colleagues among 14,259 patients undergoing CABG at 17 different cardiac centers from 2006 to 2010 (32).

The relationship between allogeneic blood transfusion and postoperative pneumonia can be partially explained by changes in immune function $(33,34)$. Longer storage of transfused blood products was also reported to be associated with an increased risk of postoperative pneumonia as well as poorer outcomes, which may be explained by the reduced oxygen-carrying capacity and transfusion-related inflammatory reactions (35-38). Specific recommendations have been provided in several clinical practice guidelines, and a restrictive transfusion strategy should be especially recommended to decrease the incidence of postoperative pneumonia and improve prognosis (39-41). Furthermore, studies aiming to identify alternative therapies or focusing on comparisons of the different outcomes between homologous and autologous transfusion may be a novel direction for future research (42).

Some other preoperative risk factors were identified in previously published studies that were not included in our model, such as cerebrovascular disease, peripheral vascular disease, weight loss, atrial fibrillation, ulcerative disease, right ventricular systolic pressure, and several laboratory values $(6,20,21,43-46)$. Numerous clinical trials have shown that 
prolonged mechanical ventilation substantially increases the risk of postoperative pneumonia $(9,22,47,48)$, which is associated with damage to the defense mechanism of the respiratory system caused by endotracheal intubation (49). The incidence of postoperative pneumonia was reported to increase by $1-3 \%$ if mechanical ventilation was extended by 1 day; thus, early extubation should be performed if conditions permit (50,51). Indeed, a significant difference in the mechanical ventilation duration existed between patients with and without postoperative pneumonia in our results, and the duration was relatively long compared with durations reported in the literature $(6,8,44,45)$, which may partly account for the high incidence of postoperative pneumonia. However, the duration of mechanical ventilation was not incorporated into our risk model because of its nature as a postoperative variable and because this parameter cannot be assessed very early. Nevertheless, as a modifiable factor, the mechanical ventilation duration could have been shorter if a more rigorous ventilation strategy had been implemented and will certainly be an important aspect that can be improved in our future clinical practice. Reintubation was also reported as a risk factor for postoperative pneumonia in several studies $(45,52,53)$, but we found that most operations were carried out after the emergence of pneumonia, which was also one of the reasons why we excluded it from our analysis.

The prediction model may play an important role in risk stratification and identification of high-risk populations. Appropriate preventive measures and specific interventions focusing on high-risk patient subsets may be more efficient. Guidelines for the management of postoperative pneumonia $(14,16)$ are available, and several measures have been reported to be effective in reducing the occurrence of postoperative pneumonia, such as subglottic secretion drainage $(54,55)$, oropharyngeal nursing with chlorhexidine (56), respiratory physiotherapy (57), silvercoated endotracheal tubes (58) and selective digestive decontamination (59). Nevertheless, some of these means are laborious, time consuming and expensive, which would certainly lead to a substantial waste of medical staff labor and resources if these techniques are applied to all patients without selection. Instead, adequate prevention and treatment targeting patients in the higher risk category identified by our risk model may be a better clinical strategy. In addition, the score can play a certain guiding role in the communication between doctors and patients, which is an important part of modern medical activities that cannot be underestimated. The most fearful thing for patients and their families is their ignorance of the condition, but a simple and intuitive risk score can help them understand the risk of postoperative pneumonia more easily and reduce their psychological stress.

\section{Limitations}

Some limitations of this study should be mentioned. Our prediction model originated from a retrospective study based on experiences in a single institution, which may restrict its wide application to other centers. Therefore, external validation should be performed to verify our conclusion before the model is conventionally used in clinical practice. Furthermore, although the ratio of sample-to-candidate variables was greater than 10 , the number of samples included in this study may be considered relatively small, and a prospective multicenter study with a large sample size is required in future work. Undeniably, a certain degree of subjectivity and variability existed in the clinical diagnosis of postoperative pneumonia despite the standardized diagnostic criteria being established before the start of the study, which may have resulted in underestimation or overestimation of our real rate of postoperative pneumonia. In addition, some variables that may affect the incidence of postoperative pneumonia, such as the operation type and drug use, were not included in our analysis. Finally, for convenient comparison and score allocation, all variables eventually included in the model were dichotomized, which may have resulted in the loss of some individual information.

\section{Conclusions}

In this study, postoperative pneumonia was a prevalent complication related to an increased risk of mortality in patients undergoing cardiac surgery. We developed and validated a prediction model for pneumonia after cardiac surgery using 13 independent risk factors and created three risk intervals based on a 32 -point risk score. The prediction rule performed well in terms of both calibration and discrimination, and we believe that it has good clinical utility as the incorporated variables are easily obtainable and the risk stratification allows better targeted interventions to reduce the incidence of postoperative pneumonia in higherrisk patients.

\section{Acknowledgments}

The authors would like to thank American fournal Experts 
for their language editing.

Funding: This work was supported by the National Natural Science Foundation of China (Grant No. 81800413).

\section{Footnote}

Reporting Checklist: The authors have completed the STROBE reporting checklist. Available at http://dx.doi. org/10.21037/jtd-20-3586

Data Sharing Statement: Available at http://dx.doi. org/10.21037/jtd-20-3586

Peer Review File: Available at http://dx.doi.org/10.21037/jtd20-3586

Conflicts of Interest: All authors have completed the ICMJE uniform disclosure form (available at http://dx.doi. org/10.21037/jtd-20-3586). The authors have no conflicts of interest to declare.

Etbical Statement: The authors are accountable for all aspects of the work in ensuring that questions related to the accuracy or integrity of any part of the work are appropriately investigated and resolved. The study was conducted in accordance with the Declaration of Helsinki (as revised in 2013). The study was approved by the Ethics Committee of Tongji Medical College of Huazhong University of Science and Technology (IORG No. IORG0003571) and individual consent for this retrospective analysis was waived.

Open Access Statement: This is an Open Access article distributed in accordance with the Creative Commons Attribution-NonCommercial-NoDerivs 4.0 International License (CC BY-NC-ND 4.0), which permits the noncommercial replication and distribution of the article with the strict proviso that no changes or edits are made and the original work is properly cited (including links to both the formal publication through the relevant DOI and the license). See: https://creativecommons.org/licenses/by-nc-nd/4.0/.

\section{References}

1. He S, Chen B, Li W, et al. Ventilator-associated pneumonia after cardiac surgery: a meta-analysis and systematic review. J Thorac Cardiovasc Surg 2014;148:3148-55.e1.
2. Tamayo E, Alvarez FJ, Martinez-Rafael B, et al. Ventilator-associated pneumonia is an important risk factor for mortality after major cardiac surgery. J Crit Care 2012;27:18-25.

3. LaPar DJ, Crosby IK, Rich JB, et al. A contemporary cost analysis of postoperative morbidity after coronary artery bypass grafting with and without concomitant aortic valve replacement to improve patient quality and cost-effective care. Ann Thorac Surg 2013;96:1621-7.

4. Greco G, Shi W, Michler RE, et al. Costs associated with health care-associated infections in cardiac surgery. J Am Coll Cardiol 2015;65:15-23.

5. Kollef $M H$. Ventilator-associated pneumonia. A multivariate analysis. JAMA 1993;270:1965-70.

6. Kinlin LM, Kirchner C, Zhang H, et al. Derivation and validation of a clinical prediction rule for nosocomial pneumonia after coronary artery bypass graft surgery. Clin Infect Dis 2010;50:493-501.

7. Klompas M. Complications of mechanical ventilation-the CDC's new surveillance paradigm. N Engl J Med 2013;368:1472-5.

8. El Solh AA, Bhora M, Pineda L, et al. Nosocomial pneumonia in elderly patients following cardiac surgery. Respir Med 2006;100:729-36.

9. Hortal J, Giannella M, Perez MJ, et al. Incidence and risk factors for ventilator-associated pneumonia after major heart surgery. Intensive Care Med 2009;35:1518-25.

10. Bonell A, Azarrafiy R, Huong V, et al. A Systematic Review and Meta-analysis of Ventilator-associated Pneumonia in Adults in Asia: An Analysis of National Income Level on Incidence and Etiology. Clin Infect Dis 2019;68:511-8.

11. Lola I, Levidiotou S, Petrou A, et al. Are there independent predisposing factors for postoperative infections following open heart surgery? J Cardiothorac Surg 2011;6:151.

12. Xiao P, Song W, Han Z. Characteristics of pulmonary infection after mitral valve repair in patients with metabolic syndrome and its relationship with blood pressure, blood glucose and blood lipid. Exp Ther Med 2018;16:5003-8.

13. Leal-Noval SR, Marquez-Vacaro JA, Garcia-Curiel A, et al. Nosocomial pneumonia in patients undergoing heart surgery. Crit Care Med 2000;28:935-40.

14. Kalil AC, Metersky ML, Klompas M, et al. Management of Adults With Hospital-acquired and Ventilator-associated Pneumonia: 2016 Clinical Practice Guidelines by the Infectious Diseases Society of America and the American Thoracic Society. Clin Infect Dis 2016;63:e61-e111.

15. Anonymous. Guidelines for the management, of adults 
with hospital-acquired, ventilator-associated, and healthcare-associated pneumonia. Am J Respir Crit Care Med 2005;171:388-416.

16. Torres A, Niederman MS, Chastre J, et al. International ERS/ESICM/ESCMID/ALAT guidelines for the management of hospital-acquired pneumonia and ventilator-associated pneumonia: Guidelines for the management of hospital-acquired pneumonia (HAP)/ ventilator-associated pneumonia (VAP) of the European Respiratory Society (ERS), European Society of Intensive Care Medicine (ESICM), European Society of Clinical Microbiology and Infectious Diseases (ESCMID) and Asociacion Latinoamericana del Torax (ALAT). Eur Respir J 2017;50:1700582.

17. Fitch ZW, Whitman GJ. Incidence, risk, and prevention of ventilator-associated pneumonia in adult cardiac surgical patients: a systematic review. J Card Surg 2014;29:196-203.

18. Duchnowski P, Hryniewiecki T, Kusmierczyk M, et al. Performance of the EuroSCORE II and the Society of Thoracic Surgeons score in patients undergoing aortic valve replacement for aortic stenosis. J Thorac Dis 2019;11:2076-81.

19. He S, Wu F, Wu X, et al. Ventilator-associated events after cardiac surgery: evidence from 1,709 patients. J Thorac Dis 2018;10:776-83.

20. Kilic A, Ohkuma R, Grimm JC, et al. A novel score to estimate the risk of pneumonia after cardiac surgery. $\mathrm{J}$ Thorac Cardiovasc Surg 2016;151:1415-20.

21. Strobel RJ, Liang Q, Zhang M, et al. A Preoperative Risk Model for Postoperative Pneumonia After Coronary Artery Bypass Grafting. Ann Thorac Surg 2016;102:1213-9.

22. Ailawadi G, Chang HL, O'Gara PT, et al. Pneumonia after cardiac surgery: Experience of the National Institutes of Health/Canadian Institutes of Health Research Cardiothoracic Surgical Trials Network. J Thorac Cardiovasc Surg 2017;153:1384-91.e3.

23. McDonald CI, Fraser JF, Coombes JS, et al. Oxidative stress during extracorporeal circulation. Eur J Cardiothorac Surg 2014;46:937-43.

24. Allou N, Bronchard R, Guglielminotti J, et al. Risk factors for postoperative pneumonia after cardiac surgery and development of a preoperative risk score* . Crit Care Med 2014;42:1150-6.

25. Anastasiadis K, Murkin J, Antonitsis P, et al. Use of minimal invasive extracorporeal circulation in cardiac surgery: principles, definitions and potential benefits. A position paper from the Minimal invasive Extra-Corporeal Technologies international Society (MiECTiS). Interact Cardiovasc Thorac Surg 2016;22:647-62.

26. Murkin JM. Monitoring and optimization of the microcirculation during CPB. J Thorac Dis 2019;11:S1489-91.

27. Möhnle P, Snyder-Ramos SA, Miao Y, et al. Postoperative red blood cell transfusion and morbid outcome in uncomplicated cardiac surgery patients. Intensive Care Med 2011;37:97-109.

28. Leal-Noval SR, Rincon-Ferrari MD, Garcia-Curiel A, et al. Transfusion of blood components and postoperative infection in patients undergoing cardiac surgery. Chest 2001;119:1461-8.

29. Horvath KA, Acker MA, Chang H, et al. Blood transfusion and infection after cardiac surgery. Ann Thorac Surg 2013;95:2194-201.

30. Crawford TC, Magruder JT, Fraser C, et al. Less Is More: Results of a Statewide Analysis of the Impact of Blood Transfusion on Coronary Artery Bypass Grafting Outcomes. Ann Thorac Surg 2018;105:129-36.

31. Likosky DS, Paone G, Zhang M, et al. Red Blood Cell Transfusions Impact Pneumonia Rates After Coronary Artery Bypass Grafting. Ann Thorac Surg 2015;100:794$800,801$.

32. LaPar DJ, Crosby IK, Ailawadi G, et al. Blood product conservation is associated with improved outcomes and reduced costs after cardiac surgery. J Thorac Cardiovasc Surg 2013;145:796-803, 803-4.

33. Tormey CA, Hendrickson JE. Transfusion-related red blood cell alloantibodies: induction and consequences. Blood 2019;133:1821-30.

34. Karsten E, Herbert BR. The emerging role of red blood cells in cytokine signalling and modulating immune cells. Blood Rev 2020;41:100644.

35. Vamvakas EC, Carven JH. Transfusion and postoperative pneumonia in coronary artery bypass graft surgery: effect of the length of storage of transfused red cells. Transfusion 1999;39:701-10.

36. Tinmouth A, Fergusson D, Yee IC, et al. Clinical consequences of red cell storage in the critically ill. Transfusion 2006;46:2014-27.

37. Roback JD, Neuman RB, Quyyumi A, et al. Insufficient nitric oxide bioavailability: a hypothesis to explain adverse effects of red blood cell transfusion. Transfusion 2011;51:859-66.

38. Hod EA, Brittenham GM, Billote GB, et al. Transfusion of human volunteers with older, stored red blood cells 
produces extravascular hemolysis and circulating nontransferrin-bound iron. Blood 2011;118:6675-82.

39. Carson JL, Guyatt G, Heddle NM, et al. Clinical Practice Guidelines From the AABB: Red Blood Cell Transfusion Thresholds and Storage. JAMA 2016;316:2025-35.

40. Ferraris VA, Ferraris SP, Saha SP, et al. Perioperative blood transfusion and blood conservation in cardiac surgery: the Society of Thoracic Surgeons and The Society of Cardiovascular Anesthesiologists clinical practice guideline. Ann Thorac Surg 2007;83:S27-S86.

41. Ferraris VA, Brown JR, Despotis GJ, et al. 2011 update to the Society of Thoracic Surgeons and the Society of Cardiovascular Anesthesiologists blood conservation clinical practice guidelines. Ann Thorac Surg 2011;91:944-82.

42. American Society of Anesthesiologists Task Force on Perioperative Blood Management. Practice guidelines for perioperative blood management: an updated report by the American Society of Anesthesiologists Task Force on Perioperative Blood Management*. Anesthesiology 2015;122:241-75.

43. Santos M, Braga JU, Gomes RV, et al. Predictive factors for pneumonia onset after cardiac surgery in Rio de Janeiro, Brazil. Infect Control Hosp Epidemiol 2007;28:382-8.

44. Topal AE, Eren MN. Risk factors for the development of pneumonia post cardiac surgery. Cardiovasc J Afr 2012;23:212-5.

45. Bouza E, Perez A, Munoz P, et al. Ventilator-associated pneumonia after heart surgery: a prospective analysis and the value of surveillance. Crit Care Med 2003;31:1964-70.

46. Duchnowski P, Hryniewiecki T, Kusmierczyk M, et al. Right ventricular systolic pressure as a predictive factor for postoperative pneumonia in patients with valvular heart disease. Kardiol Pol 2019;77:969-71.

47. Hortal J, Munoz P, Cuerpo G, et al. Ventilator-associated pneumonia in patients undergoing major heart surgery: an incidence study in Europe. Crit Care 2009;13:R80.

48. Sheng W, Xing QS, Hou WM, et al. Independent risk factors for ventilator-associated pneumonia after cardiac surgery. J Invest Surg 2014;27:256-61.

49. Tobin M, Manthous C. Mechanical Ventilation. Am J Respir Crit Care Med 2017;196:P3-P4.

50. Bouadma L, Wolff M, Lucet JC. Ventilator-associated pneumonia and its prevention. Curr Opin Infect Dis
2012;25:395-404.

51. Papazian L, Klompas M, Luyt CE. Ventilator-associated pneumonia in adults: a narrative review. Intensive Care Med 2020;46:888-906.

52. Halpin L, Henry L, Szelkowski L, et al. Ventilatorassociated pneumonia among cardiac surgery patients: what can we do for prevention? J Nurs Care Qual 2013;28:345-51.

53. Gao F, Yang LH, He HR, et al. The effect of reintubation on ventilator-associated pneumonia and mortality among mechanically ventilated patients with intubation: A systematic review and meta-analysis. Heart Lung 2016;45:363-71.

54. Pozuelo-Carrascosa DP, Herraiz-Adillo A, Alvarez-Bueno C, et al. Subglottic secretion drainage for preventing ventilator-associated pneumonia: an overview of systematic reviews and an updated meta-analysis. Eur Respir Rev 2020;29:190107.

55. Bouza E, Perez MJ, Munoz P, et al. Continuous aspiration of subglottic secretions in the prevention of ventilatorassociated pneumonia in the postoperative period of major heart surgery. Chest 2008;134:938-46.

56. Bardia A, Blitz D, Dai F, et al. Preoperative chlorhexidine mouthwash to reduce pneumonia after cardiac surgery: A systematic review and meta-analysis. J Thorac Cardiovasc Surg 2019;158:1094-100.

57. Katsura M, Kuriyama A, Takeshima T, et al. Preoperative inspiratory muscle training for postoperative pulmonary complications in adults undergoing cardiac and major abdominal surgery. Cochrane Database Syst Rev 2015:CD010356.

58. Kollef MH, Afessa B, Anzueto A, et al. Silver-coated endotracheal tubes and incidence of ventilator-associated pneumonia: the NASCENT randomized trial. JAMA 2008;300:805-13.

59. Schnabel RM, Scholte JB, Van Der Velden KE, et al. Ventilator-associated pneumonia rates after introducing selective digestive tract decontamination. Infect Dis (Lond) 2015;47:650-3.

Cite this article as: Wang D, Huang X, Wang H, Le S, Yang H, Wang F, Du X. Risk factors for postoperative pneumonia after cardiac surgery: a prediction model. J Thorac Dis 2021;13(4):23512362. doi: $10.21037 /$ jtd-20-3586 


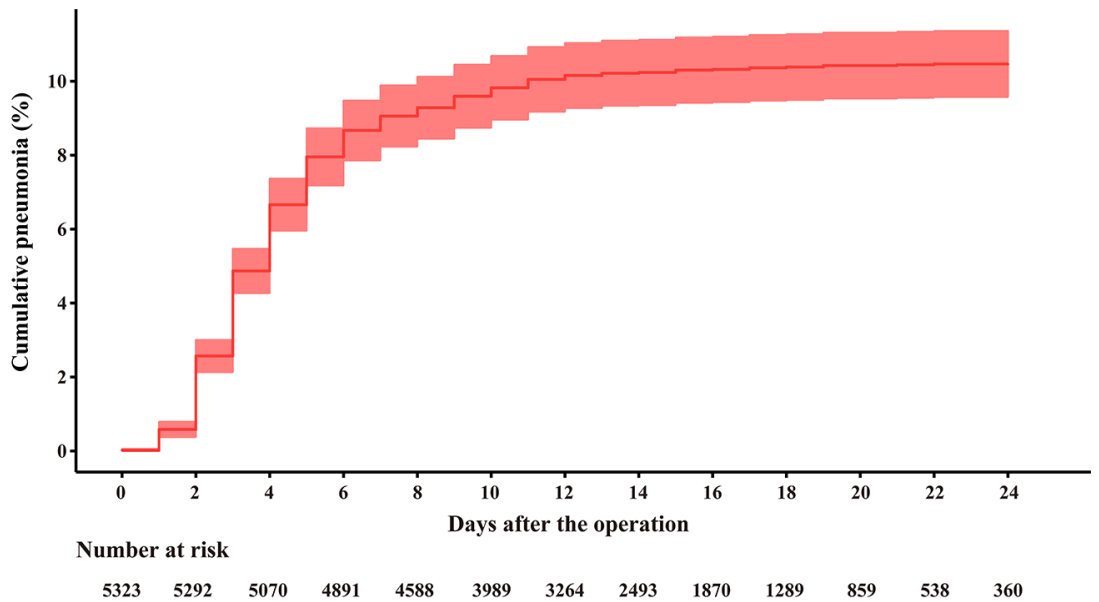

Figure S1 Kaplan-Meier curve showing the cumulative number and probability of patients with postoperative pneumonia over time. Pneumonia occurred in 530 patients $(9.96 \%)$ and $87 \%$ developed in the first postoperative week, with a median time of $4(2,5)$ days.

Table S1 Postoperative variables in patients with and without postoperative pneumonia after cardiac surgery

\begin{tabular}{|c|c|c|c|c|}
\hline Variables & $\begin{array}{l}\text { Without postoperative } \\
\text { pneumonia, } n=4,793(\%)\end{array}$ & $\begin{array}{c}\text { With postoperative } \\
\text { pneumonia, } n=530(\%)\end{array}$ & Odds ratio $(95 \% \mathrm{Cl})$ & $P$ value \\
\hline Mechanical ventilation (hours) & $22(18,39)$ & $125(69,223)$ & $1.036(1.033,1.039)$ & $<0.001$ \\
\hline Hospital stay (days) & $14(11,18)$ & $26(20,34)$ & $1.171(1.157,1.186)$ & $<0.001$ \\
\hline Reintubation & $50(1.04)$ & $197(37.17)$ & $56.118(40.359,78.032)$ & $<0.001$ \\
\hline Mortality & $31(0.65)$ & $136(25.66)$ & $52.166(35.887,75.831)$ & $<0.001$ \\
\hline
\end{tabular}

Abbreviations: $\mathrm{Cl}$, confidence interval; ICU, intensive care unit. 